\title{
Indicadores de actividad durante cinco años en un servicio de urgencias de atención voluntaria de un hospital psiquiátrico mexicano
}

\author{
Alejandro Molina-López, ${ }^{1}$ María Cristina López-Pedraza, ${ }^{2}$ Nadia Morales-Gordillo, ${ }^{3}$ \\ Martha Patricia Ontiveros Uribe, ${ }^{4}$ Ana Fresán-Orellana ${ }^{5}$
}

Artículo original

\section{SUMMARY \\ Background}

The demand for Psychiatry Emergency Services (PES) has increased during the last years. There is little knowledge about the activity indicators of PES at Mexican psychiatric hospitals. It is necessary to study the activities of these PES, especially the ones which work through voluntary care and with no emergency severity assessment procedure (Triage) before consultation.

\section{Objective}

To describe and compare the activity indicators of a PES within a psychiatric hospital in Mexico City during five years. This hospital offered only voluntary care with no Triage procedure.

\section{Material and method}

The database of all registered PES visits from January $1^{\text {st }}, 2004$, to December $31^{\text {th }}, 2008$, was analysed. We determined the overall number of consultations and relative frequencies by trimester, semester, year and five years. After this, indicators were broken down using service variables and psychiatric diagnosis according to the ICD-10; they were then compared with each other.

\section{Results}

A total of $n=41058$ consultations were attended during five years, showing an enhancement of $14.8 \%$ in the overall PES activity. We observed a significant enhancement of patients' proportion with no compliance to outpatient treatment as well as more night schedule visitations, while references and admission proportions decreased more than $4 \%$. This suggests a "snow ball-like" progressive enhancement of frequent patients of PES with non-urgent conditions. We did not find any changes in psychiatric diagnosis proportion during the study, but there was a significant $9.69 \%$ increase in the overall activity of the first semester compared with the second one.

\section{Discussion}

Voluntary care with no Triage procedure tends to enhance the influx of frequent patients with non-urgent conditions, leading to PES overcrowding and disfavoring urgent conditions, specially during the first semester of each year.

Key words: Activity indicator, psychiatric emergency, triage, service utilization.

\section{RESUMEN}

Introducción

Los Servicios de Urgencias Psiquiátricas (SUP) han reportado incremento en su demanda en los últimos años. Se desconoce sobre los indicadores de actividad en SUP en hospitales psiquiátricos mexicanos. Se necesitan estudios que describan la actividad de estos servicios, especialmente los de atención voluntaria y sin procedimiento de valoración de la gravedad de las urgencias (triage) previo a la consulta.

\section{Objetivos}

Describir y comparar los Indicadores de Actividad en un SUP de un hospital psiquiátrico de la Ciudad de México durante cinco años, en el que se ofreció atención voluntaria sin procedimiento de triage.

\section{Material y métodos}

Se analizó una base de datos del SUP de todas las consultas otorgadas del 10 de enero de 2004 al 31 de diciembre de 2008 . Se determinaron el total de consultas y las frecuencias relativas trimestral, semestral, anual y quinquenal desglosados por variables de utilización de servicio y diagnóstico psiquiátrico según la CIE-10.

\section{Resultados}

Se atendieron un total de $\mathrm{n}=41058$ consultas durante el quinquenio, encontrando incremento de $14.8 \%$ en la actividad global en el

\footnotetext{
Servicio de Atención Psiquiátrica Continua, Instituto Nacional de Psiquiatría Ramón de la Fuente Muñiz, INPRFM.

Programa de Voluntad Anticipada, Secretaría de Salud del Distrito Federal.

Servicio de Psicofarmacología del Desarrollo, Hospital Psiquiátrico Infantil Juan N. Navarro.

Subdirección de Hospital y Atención Psiquiátrica Continua, INPRFM.

Departamento de Epidemiología Psiquiátrica, INPRFM.
}

Correspondencia: Alejandro Molina-López. Instituto Nacional de Psiquiatría Ramón de la Fuente Muñiz. Calz. México-Xochimilco 101, San Lorenzo Huipulco, Tlalpan, 14370, México, DF., E-mail: amolinal@imp.edu.mx

Recibido primera versión: 31 julio de 2014. Segunda versión: 25 de septiembre de 2014. Aceptado: 07 de octubre de 2014. 
período. Se observó un incremento significativo de la proporción de pacientes sin adherencia a consulta externa así como mayor afluencia en el turno nocturno, mientras que la proporción de referencias y hospitalizaciones disminuyó más del $4 \%$, sugiriendo aumento "en bola de nieve" de usuarios frecuentadores con condiciones no urgentes. No se encontraron cambios en la proporción de los diagnósticos psiquiátricos a lo largo del tiempo, pero hubo un aumento significativo del $9.69 \%$ en la actividad global en el primer semestre respecto del segundo.

\section{Discusión}

La atención voluntaria sin procedimiento de triage tiende a generar mayor afluencia de pacientes frecuentadores con condiciones no urgentes, sobresaturando el servicio y desfavoreciendo las condiciones urgentes, especialmente durante el primer semestre del año.

Palabras clave: Indicador de actividad, urgencia psiquiátrica, triage, utilización de servicios.

\section{INTRODUCCION}

La Psiquiatría de Urgencias ha adquirido gran importancia en las últimas décadas. En los Estados Unidos, tras la desinstitucionalización y la búsqueda de la reincorporación de los pacientes psiquiátricos a la comunidad, miles de trastornos mentales agudos son actualmente enviados a hospitales generales, siendo los servicios de urgencias el primer punto de entrada para dichos pacientes. ${ }^{1}$ Se ha estimado que hasta un $30 \%$ de los usuarios que tienen primer contacto con los servicios de salud mental acuden a un servicio de urgencias, y se ha visto un incremento de hasta $130 \%$ del total de consultas en los Servicios de Urgencias Psiquiátricas (SUP) en los últimos años. ${ }^{2}$

Los SUP cumplen con múltiples funciones no sólo con el paciente, sino también con la comunidad, ya que los SUP frecuentemente colaboran con otros proveedores de servicios de emergencias como médicos, paramédicos y enfermeras, así como con servicios de salud mental comunitaria, en tribunales judiciales y en escuelas de todos los niveles. ${ }^{3}$ Se ha recalcado la importancia de los SUP en la academia y la investigación, ${ }^{4}$ y su participación en emergencias complejas y desastres. ${ }^{5}$ Además, la decisión de admitir o referir a un paciente psiquiátrico dentro de una sala de urgencias tiene implicaciones médicas y económicas de alto alcance. ${ }^{6}$ Así mismo se ha visto que cuando se implementan programas en los SUP consistentes en intervención en crisis en pacientes deprimidos, existe una disminución significativa en las hospitalizaciones psiquiátricas ${ }^{7}$ y disminuyen los costos individuales, comunitarios y gubernamentales. ${ }^{8}$ Además, los SUP son, en ocasiones, la única oportunidad para estimar la adherencia al tratamiento, la red de apoyo social y el pronóstico de los usuarios que demandan la consulta, ${ }^{9,10}$ lo cual puede representar una oportunidad de brindar psicoeducación, afianzar el apego al tratamiento o validar una opinión médica previa. ${ }^{11}$

A pesar de que muchos autores alrededor del mundo han publicado sobre servicios de urgencias y salud mental, existen pocos estudios relacionados con los SUP en el caso particular de México. Algunos estudios han reportado prevalencias de trastornos psiquiátricos en los servicios de urgencias de hospitales generales. ${ }^{12}$ Un número importante de estudios mexicanos se han enfocado en el papel que des- empeña el alcohol en los motivos por los que se solicita consulta de urgencias, ${ }^{13-15}$ en la prevalencia de ideación suicida en usuarios, ${ }^{16}$ así como la etnografía de la atención de los pacientes abusadores de alcohol en un servicio de urgencias. ${ }^{17}$ Algunos servicios de urgencias de hospitales generales mexicanos han participado en estudios multicéntricos, los cuales han descrito la prevalencia de los trastornos mentales en población usuaria de servicios de urgencias de hospitales generales. ${ }^{18-20}$ Aunque estos estudios son muy valiosos, los resultados obtenidos a partir de servicios de urgencias de hospitales generales no pueden interpretarse igual que en los servicios de urgencias de hospitales psiquiátricos, ya que la percepción de la enfermedad mental es completamente diferente en ambos tipos de hospital entre pacientes, familiares y proveedores de servicios de salud..$^{21,22}$

Para cuantificar la utilización de un servicio de salud, incluyendo un servicio de urgencias, el método más utilizado es con base en indicadores, que en el área de la salud pueden ser de dos tipos: de actividad y de calidad. ${ }^{23}$ Los indicadores que describen cuantitativamente la utilización de un servicio por unidad de tiempo son los indicadores de actividad, que expresan el total de atenciones brindadas durante un período de tiempo a una población específica. Los indicadores de actividad pueden representarse de dos formas: a) Número de consultas por unidad de tiempo y b) Frecuencia relativa por unidad de tiempo (generalmente por años). El primero refleja la sumatoria de todas las consultas a lo largo de un período determinado; el segundo refleja el número de consultas por tiempo determinado en relación con una población de cobertura según censos o referentes. ${ }^{24}$

Los indicadores de actividad pueden reflejar el aumento o disminución de la demanda de los servicios al compararse entre sí a lo largo del tiempo, pudiéndose considerar una representación indirecta del impacto de los servicios de los programas de salud mental existentes, ${ }^{25}$ ya que la asistencia a los servicios de urgencias y la adherencia a los servicios ambulatorios son actividades muchas veces complementarias, debido a que la demanda de los servicios de urgencias depende en mucho de la cobertura e implementación de servicios de salud mental a nivel de la comunidad. ${ }^{26,27}$

Un procedimiento frecuente en los servicios de urgencias es el triage. El término "triage" es un anglicismo prove- 
niente del francés "triere" que significa escoger, entresacar, categorizar. Se trata de un procedimiento para la categorización de la gravedad de las urgencias con el objetivo de priorizar las más graves por encima de las menos graves. ${ }^{28}$ Se conoce poco sobre los efectos con la utilización o la falta de un procedimiento de triage en un servicio de urgencias psiquiátricas, especialmente si se toma en cuenta que la actividad de un servicio a otro puede ser extremadamente variable. $^{29}$

Teniendo en consideración la falta de estudios sobre la utilización de servicios de urgencias en hospitales psiquiátricos en México, realizamos el presente trabajo que tuvo como objetivo describir y comparar los indicadores de actividad para diferentes períodos en un servicio de urgencias psiquiátricas mexicano durante los años 2004 a 2008, en el que aún no se había implementado un procedimiento de clasificación de urgencias psiquiátricas (triage), y en el que el único criterio de atención era la solicitud de atención voluntaria por parte de los usuarios que consideraron ameritar una consulta de urgencias durante el período de estudio.

\section{MATERIAL Y METODO}

\section{Sujetos}

Se incluyeron todos los registros de los usuarios que solicitaron voluntariamente una consulta en el Servicio de Urgencias del Instituto Nacional de Psiquiatría Ramón de la Fuente Muñiz (INPRFM) en el período comprendido del 1 de enero de 2004 al 31 de diciembre de 2008. Se excluyeron los registros de los usuarios que no completaron la consulta de urgencias, que la rechazaron, o que expresamente negaron su consentimiento para poder utilizar sus datos con fines bio-estadísticos. Posteriormente se elaboró una base de datos institucional con el programa Microsoft Excel basada en los censos estadísticos diarios del servicio de urgencias, los cuales se obtuvieron directamente de la nota de valoración médica de cada paciente.

\section{Institución}

El INPRFM forma parte de los Institutos Nacionales de Salud. El área designada para el servicio de urgencias se estableció desde el mes de marzo del año 2000; consiste en un área de aproximadamente $110 \mathrm{~m}^{2}$ que incluye: sala de observación con dos camas, un módulo de enfermería, dos sanitarios (para personal y para pacientes), cuatro consultorios y una oficina para el médico adscrito al servicio. Cuenta con una sala de espera situada afuera del servicio sobre un pasillo que conecta a la Consulta Externa con el área de Hospitalización. El número de consultorios aumentó, pues contaba con un solo consultorio en el año 2000, posteriormente el número de consultorios llegó a dos en 2002, a tres en 2004, a cuatro en 2006 y a cinco en 2008, lo cual se hizo redistribuyendo los espacios, permaneciendo constante el área general del servicio.

\section{Procedimiento de atención}

Los usuarios que acudieron al Servicio de Urgencias durante los años 2004 a 2008 se presentaron en forma voluntaria al servicio. Cada usuario fue enviado por el Módulo de Vigilancia al Módulo de Registro de pacientes, donde se registraba electrónicamente $\mathrm{y}$, en su caso, se cubría el coste de la consulta en las cajas. En caso de no contar con posibilidad de pago el servicio de trabajo social autorizaba la postergación de éste, no siendo el motivo económico un impedimento para la atención. Cada usuario se presentó en el servicio donde la enfermera le tomaba los signos vitales, y posteriormente tenía que esperar la consulta un tiempo variable, siendo el único criterio de priorización y tiempo de espera la demanda corriente y la saturación del servicio y la mayor o menor disponibilidad de médicos en el momento de solicitar atención. Hasta el último trimestre del año 2008 no se aplicó ningún procedimiento de clasificación de gravedad de urgencias psiquiátricas (triage), por lo que todas las condiciones se valoraron según la necesidad subjetiva de cada usuario de recibir atención inmediata de urgencias y según los recursos humanos disponibles en los diferentes momentos.

\section{Indicadores de actividad}

Se calcularon de acuerdo a los siguientes criterios: a) Total de consultas (número de consultas otorgadas por período de tiempo) y b) Frecuencia relativa (cociente del número de consultas por período de tiempo entre el referente de la población cubierta por el servicio, por cada 100000 habitantes). El referente de población cubierta se consideró como el promedio entre 2005 y 2010 de la población total reportada por el Instituto Nacional de Estadística, Geografía e Historia de los Estados de México, Morelos y el Distrito Federal, representando el referente estimado de cobertura para este estudio la sumatoria de 25072740 habitantes. ${ }^{30}$

\section{Utilización del servicio y diagnóstico psiquiátrico}

Los registros de la base de datos se desglosaron de acuerdo a dos grupos de variables registradas en la base de datos: a) Utilización del servicio y b) Diagnóstico psiquiátrico. Las variables de utilización del servicio fueron: Contacto institucional de primera vez o subsecuente, adherencia a citas en consulta externa, motivo del usuario que movilizó la consulta y referencia o derivación. Los diagnósticos psiquiátricos fueron capturados por su código según la Décima Edición de la Clasificación Internacional de Enfermedades (CIE-10). 


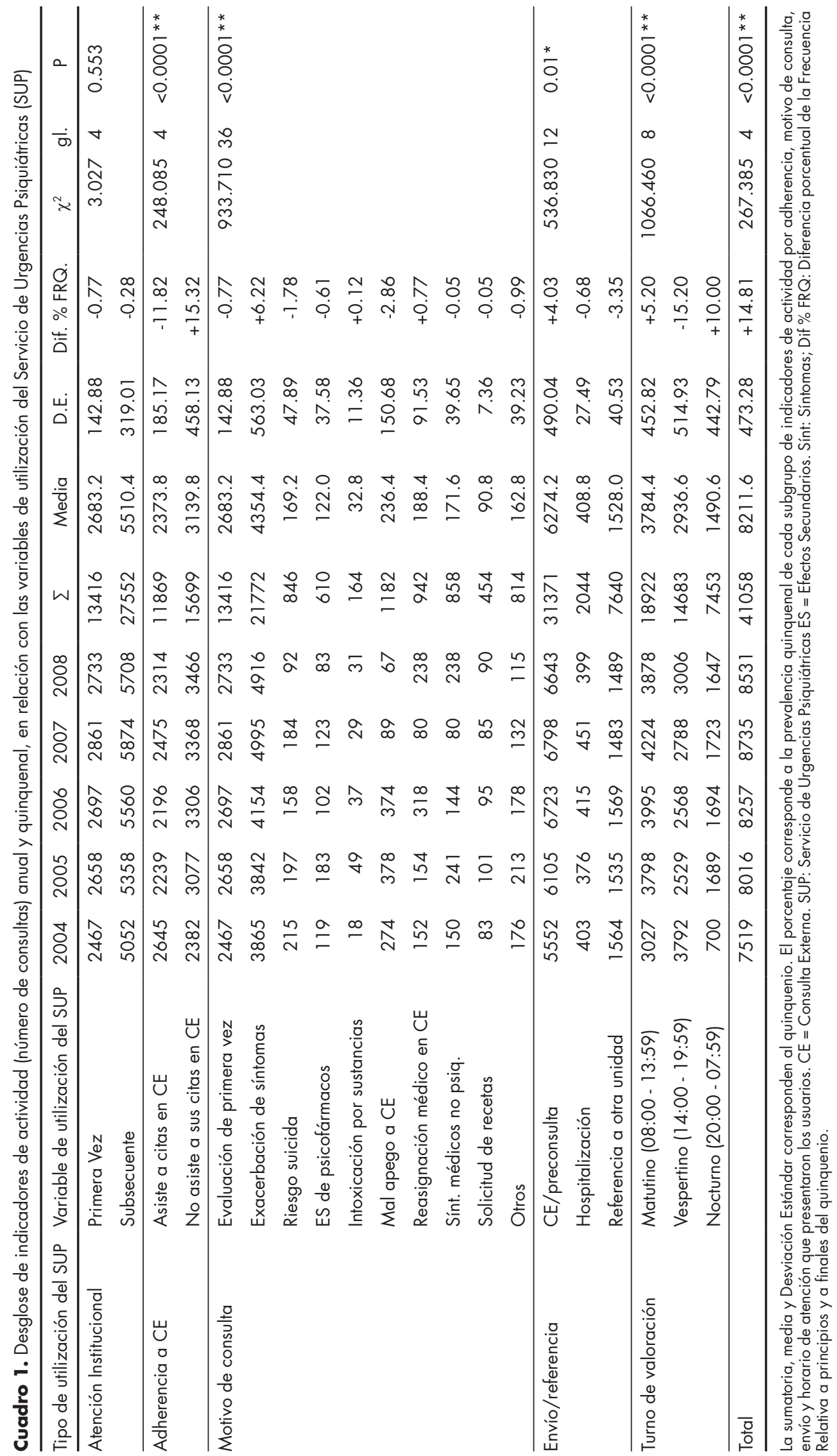


La base de datos utilizada para este estudio no contó con las variables de género, edad o riesgo suicida.

\section{Cambios de los indicadores a lo largo del tiempo}

Para confirmar las observaciones en la bibliografía de que la afluencia de urgencias psiquiátricas y ciertos diagnósticos psiquiátricos pueden tener un patrón cíclico de estacionalidad o aumento de demanda, ${ }^{31}$ los indicadores de actividad se estudiaron anual, trimestral (cuatro trimestres por año por cinco años) y semestral (dos semestres por año, por cinco años). Se compararon los indicadores inter-trimestres, inter-semestres e inter trimestre-semestre. Se calcularon los cambios porcentuales por semestre de cada variable para detectar cualquier cambio de los patrones de utilización de

Cuadro 2. Frecuencia relativa trimestral y semestral durante el quinquenio

\begin{tabular}{|c|c|c|c|c|c|c|c|c|c|c|}
\hline Diagnóstico CIE-10 & $\begin{array}{c}\text { Ene } \\
\text { a } \\
\text { Mar }\end{array}$ & $\begin{array}{c}\mathrm{Abr} \\
\mathrm{a} \\
\text { Jun }\end{array}$ & $\begin{array}{c}\text { Jul } \\
\text { a } \\
\text { Sep } \\
\end{array}$ & $\begin{array}{c}\text { Oct } \\
a \\
\text { Dic } \\
\end{array}$ & $\begin{array}{l}\text { ler } \\
\text { sem }\end{array}$ & $\begin{array}{l}2 \text { do } \\
\text { sem }\end{array}$ & $\begin{array}{c}\text { Dif } \\
\text { porcentual } \\
1 \text { y } 2 \text { sem }\end{array}$ & Comparación & $\begin{array}{l}\text { Actividad } \\
\text { global } \\
\text { estadística }\end{array}$ & $\mathrm{p}$ \\
\hline Demencias & 0.267 & 0.235 & 0.199 & 0.291 & 0.502 & 0.491 & 2.381 & & & \\
\hline Delirium & 1.918 & 1.268 & 1.312 & 1.360 & 3.187 & 2.672 & 16.145 & Inter-trimestre: & & \\
\hline Otros trastornos orgánicos & 0.052 & 0.060 & 0.116 & 0.144 & 0.112 & 0.259 & -132.140 & Trim. 1 - Trim. 2 & $t=0.5430$ & 0.591 \\
\hline Abuso/dependencia agudo & 1.165 & 1.232 & $1.300 £$ & 0.921 & 2.397 & 2.222 & 7.321 & Trim. 1 - Trim. 3 & $t=1.4340$ & 0.162 \\
\hline Abuso/dependencia crónico & 0.347 & 0.363 & 0.255 & 0.319 & 0.709 & 0.574 & 19.101 & Trim. 1 - Trim. 4 & $t=2.9730$ & $0.006 * *$ \\
\hline Esquizofrenia aguda & 0.463 & 0.542 & 0.275 & 0.331 & 1.005 & 0.606 & 39.680 & Trim. 2 - Trim. 4 & $t=2.7830$ & $0.009 * *$ \\
\hline Esquizofrenia crónica & 3.091 & 2.888 & $2.931 £$ & 2.792 & 5.978 & 5.723 & 4.269 & & & \\
\hline T. delirantes primarios & 0.307 & 0.291 & 0.215 & 0.183 & 0.598 & 0.399 & 33.330 & & & \\
\hline Otros T. psicóticos & 0.012 & 0.028 & 0.000 & 0.012 & 0.040 & 0.012 & 70.000 & & & \\
\hline Trastorno depresivo mayor & 16.090 & $16.91 €$ & 16.640 & 14.760 & 33.000 & 31.400 & 4.858 & & & \\
\hline Trastorno bipolar & 3.661 & 3.243 & 3.015 & 2.912 & 6.903 & 5.927 & 14.153 & & & \\
\hline Trastornos distímicos & 0.335 & 0.335 & 0.271 & 0.319 & 0.670 & 0.590 & 11.904 & & & \\
\hline Trastorno de angustia & 0.136 & 0.140 & 0.092 & 0.084 & 0.175 & 0.175 & 36.231 & Trim. - Sem. & & \\
\hline T. Ansiedad generalizada & 7.143 & 6.481 & 5.376 & 5.695 & 13.620 & 11.070 & 18.735 & Trim. 1 - Sem. 1 & $t=-2.3850$ & $0.024^{*}$ \\
\hline T. obsesivo compulsivo & 1.041 & 0.957 & 0.850 & 0.885 & 1.998 & 1.735 & 13.174 & Trim. 2 - Sem. 1 & $t=-2.1730$ & $0.038^{*}$ \\
\hline Reacciones graves al estrés & 0.830 & 0.722 & 0.658 & 0.698 & 1.551 & 1.356 & 12.596 & Trim. 3 - Sem. 1 & $t=-2.4990$ & $0.018^{*}$ \\
\hline T. alimentario & 0.618 & 0.447 & 0.511 & 0.391 & 1.065 & 0.901 & 15.356 & Trim. 4 -Sem. 1 & $t=-2.3160$ & $0.028^{*}$ \\
\hline T. sexualidad & 0.076 & 0.088 & 0.080 & 0.076 & 0.163 & 0.155 & 4.878 & Trim. 1 -Sem. 2 & $t=-2.5440$ & $0.017^{*}$ \\
\hline T. sueño & 0.016 & 0.012 & 0.004 & 0.012 & 0.028 & 0.016 & 42.857 & Trim. 2 - Sem. 2 & $t=-2.3932$ & $0.023^{*}$ \\
\hline T. somatoformes & 0.000 & 0.000 & 0.000 & 0.012 & 0.000 & 0.012 & 0.000 & Trim. 3 - Sem. 2 & $t=-2.4812$ & $0.019 *$ \\
\hline Cluster A & 0.008 & 0.028 & 0.012 & 0.004 & 0.036 & 0.016 & 55.556 & Trim. 4 -Sem. 2 & $t=-2.3182$ & $0.028^{*}$ \\
\hline Cluster B & 1.344 & 1.169 & 1.033 & 1.149 & 2.513 & 2.181 & 13.174 & Sem. 1 -Sem. 2 & $t=2.7830$ & 0.009 * * \\
\hline Cluster C & 0.032 & 0.052 & 0.020 & 0.016 & 0.083 & 0.000 & 57.142 & & & \\
\hline T. de la conducta & 0.191 & 0.183 & 0.168 & 0.160 & 0.375 & 0.327 & 12.765 & & & \\
\hline T.D.A.H. & 0.160 & 0.263 & 0.076 & 0.128 & 0.442 & 0.203 & 51.886 & Diagnóst. - Trim.: & & \\
\hline T. afectivos adolesc. & 0.016 & 0.016 & 0.028 & 0.004 & 0.032 & 0.032 & 0.000 & Diagnóst. - Trim. 1 & $F=1.4930$ & 0.219 \\
\hline Otros T. adolescencia & 0.080 & 0.040 & 0.056 & 0.072 & 0.120 & 0.128 & -6.660 & Diagnóst. - Trim. 2 & $F=1.4600$ & 0.231 \\
\hline T. médico no psiquiátrico & 0.283 & 0.215 & 0.327 & 0.148 & 0.499 & 0.475 & 4.800 & Diagnóst. - Trim. 3 & $F=1.4500$ & 0.234 \\
\hline T. psiq. sec. C. médica & 3.227 & 3.729 & 3.849 & 2.999 & 6.955 & 6.848 & 1.548 & Diagnóst. - Trim. 4 & $F=1.4700$ & 0.227 \\
\hline Sin diagnóstico & 0.427 & 0.606 & 0.554 & 0.455 & 1.032 & 1.009 & 2.317 & Diagnóst. - Sem. 1 & $F=1.4770$ & 0.224 \\
\hline Total & 43.340 & 42.540 & 40.220 & 37.330 & 85.880 & 77.550 & 9.696 & Diagnóst. - Sem. 2 & $F=1.4590$ & 0.231 \\
\hline
\end{tabular}

Las cifras representan la tasa de actividad del servicio de urgencias por cada 100,000 habitantes según la población referente de cobertura. La diferencia porcentual se obtuvo del porcentaje acumulado por semestre entre los primeros y segundos semestres durante el quinquenio. Los porcentajes negativos implican mayor actividad en el segundo semestre en relación con el primero. 


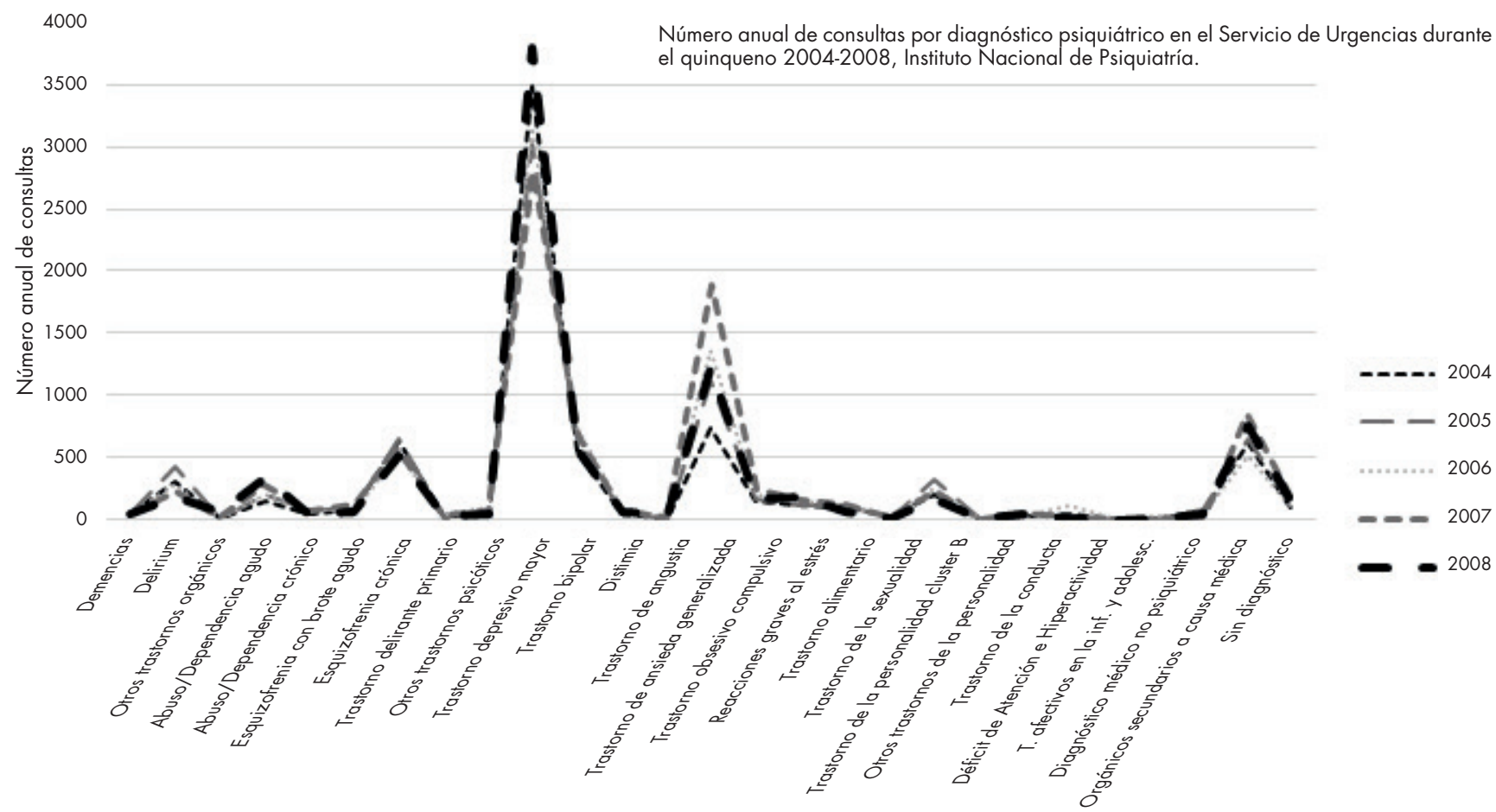

Figura 1. Los diagnósticos se asignaron de acuerdo a los registros clínicos de los pacientes generados de las consultas de urgencias.

los servicios o de la prevalencia de los diagnósticos psiquiátricos a lo largo de diferentes períodos de tiempo.

\section{Consideraciones éticas}

Se consideraron los principios de la Declaración de Helsinski sobre la Investigación en seres humanos. ${ }^{32}$ Los registros de este estudio se obtuvieron en forma retrospectiva a partir de las libretas donde se asienta la información de las notas médicas realizadas posteriormente a la atención de los usuarios del servicio de urgencias, quienes por procedimiento otorgaron su consentimiento informado en el que además de autorizar una atención voluntaria, el usuario autoriza que la información se pueda utilizar en forma anónima con fines bio-estadísticos. La confidencialidad y la privacidad de los usuarios se cuidó utilizando variables en forma numérica de tal forma que no fuese posible la identificación de los mismos.

\section{Análisis estadístico}

Para el análisis descriptivo se utilizaron medidas de tendencia central, porcentual, dispersión y sumatoria quinquenal de los indicadores de actividad anual. Para el análisis comparativo entre indicadores de actividad y utilización del servicio se utilizó prueba de chi cuadrada. Para el análisis de los cambios entre diagnósticos psiquiátricos por trimestre, semestre y quinquenio se utilizó la prueba $t$ y ANOVA de un factor; se utilizó el paquete estadístico para Ciencias Sociales (SPSS) Versión 22.0.

\section{RESULTADOS}

\section{Número total anual y quinquenal de consultas de urgencias}

Se otorgó un total de $\mathrm{n}=41160$ consultas de urgencias en el transcurso de los cinco años, en las cuales $n=102$ personas se negaron a recibir atención en forma voluntaria, resultando una muestra analizada de $\mathrm{n}=41058$ consultas. El año 2007 resultó ser el de mayor actividad del quinquenio alcanzando $n=8735$ consultas. La media quinquenal fue de $8211.6 \pm$ 473.28 consultas. Se observó un aumento progresivo en las consultas de urgencias de 2004 a 2007, presentándose un incremento anual de $6.61 \%$ en 2005 , de $3.01 \%$ en 2006 y de $5.79 \%$ en 2007, respecto del año anterior, en tanto que 2008 mostró un decremento del $2.33 \%$ respecto del 2007. Hubo un incremento global quinquenal del $14.81 \%$.

La mayoría de las consultas fueron subsecuentes $(67.11 \%$, $\mathrm{n}=27552)$, de las cuales $\mathrm{n}=15699$ (38\% de la muestra total $\mathrm{y}$ $56.98 \%$ de la muestra de subsecuentes) no tenían adecuada adherencia a las citas en consulta externa. El 53.03\% ( $n=21$ 772) acudieron a urgencias debido a una exacerbación de sus síntomas. Más de las tres cuartas partes de la muestra ( $\mathrm{n}=31$ 317, $76.41 \%$ ) fue enviada a servicios ambulatorios de la institución (Consulta externa o Preconsulta). Se hospitalizaron un total de $\mathrm{n}=2044$ pacientes, representando el $4.98 \%$ de la muestra total y generando una media de $408 \pm 27.49$ hospitalizaciones por año. Se observó una mayor proporción de consultas otorgadas en el turno matutino respecto del vespertino y nocturno. 


\section{Cambios en la frecuencia relativa anual y quinquenal}

Las variables de utilización de servicio fueron convertidas a indicadores de frecuencia relativa trimestral y semestral, analizando las diferencias porcentuales al inicio (2004) y al final (2008) del quinquenio. El cuadro 2 resume los cambios en las frecuencias relativas por trimestre y semestre. Se encontraron diferencias no significativas en las proporciones iniciales y finales de consultas de primera vez y subsecuentes $\left(\chi^{2}=3.027, \mathrm{gl}=4, \mathrm{p}=0.553\right)$. Se observó una disminución significativa del $11.82 \%$ de usuarios subsecuentes con adherencia a consulta externa, y aumento del $15.32 \%$ en la proporción de usuarios subsecuentes sin adherencia a Consulta externa al final del quinquenio $\left(\chi^{2}=248.085\right.$, gl=4, $\left.\mathrm{p}<0.0001\right)$, así como un aumento del $4.03 \%$ en el envío a Consulta externa y una disminución de las referencias a otras instituciones del 3.35\% $\left(\chi^{2}=536.83, \mathrm{gl}=12, \mathrm{p}=0.01\right)$. Se observó un incremento quinquenal del $10 \%$ en las consultas del turno nocturno $\left(\chi^{2}=1066.46, \mathrm{gl}=8, \mathrm{p}<0.0001\right)$.

\section{Diagnóstico psiquiátrico en el servicio de urgencias}

Se desglosaron cada uno de los diagnósticos psiquiátricos que presentó la muestra por código según la Sección F de la CIE-10. La figura 1 esquematiza las distribuciones de diagnósticos por año. El diagnóstico más frecuente fue el de tras-

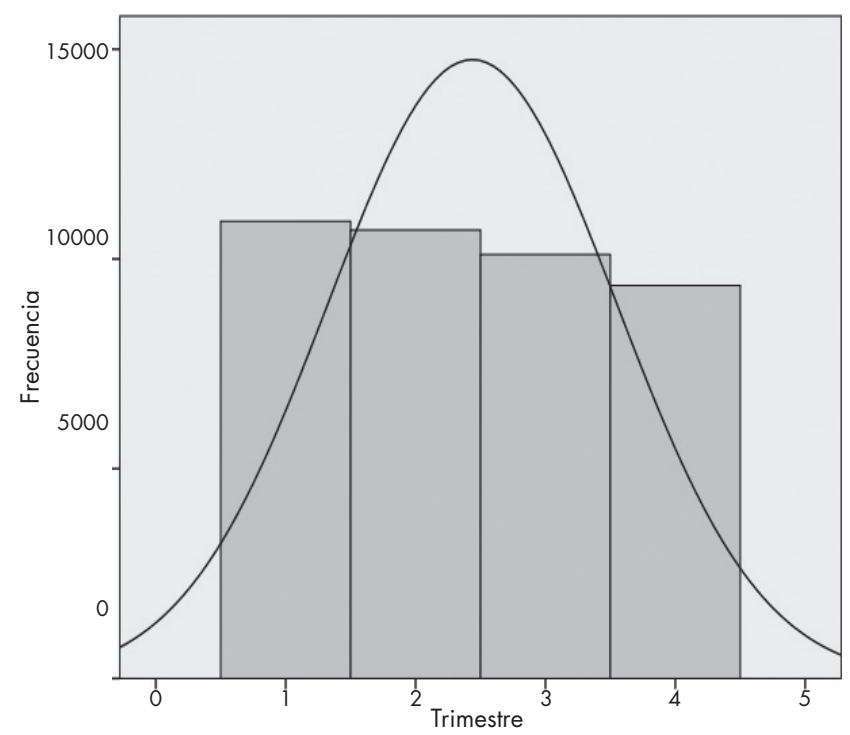

Figura 2. Actividad global inter-trimestral del Servicio de Urgencias en cico años. Distribución y curva de frecuencia de consultas de urgencias por trimestre. Se presentan las sumatorias de consultas desglosadas en cada uno de los cuatro trimestres $(1$ = enero a marzo; 2 = abril a junio; 3 = julio a septiembre; 4 = octubre a diciembre) durante 2004 a 2008. Se calculó sesgo $=0.77$ (Error estándar $=$ 0.12 ) y Curtosis $=-1.338$ (Error estándar $=0.24$ ), correspondiente a una distribución sesgada a la derecha y platicúrtica. torno depresivo mayor (prevalencia de 39.61\%,n=19.262) con una media de $3252.4 \pm 393.35$ consultas anuales. El segundo diagnóstico más frecuente fue el trastorno de ansiedad generalizada $(15.34 \%, n=6306)$, con media de $1262.2 \pm 417.79$ consultas anuales. En tercer lugar se encontraron los trastornos psiquiátricos secundarios a causa médica $(8.21 \%, \mathrm{n}=3 \mathrm{370})$ seguido del trastorno bipolar $(7.72 \%, n=3170)$, esquizofrenia paranoide crónica $(7.0 \%, \mathrm{n}=2 \mathrm{876})$, delirium secundario a causa médica $(3.46 \%, n=1419)$, seguido de los trastornos adictivos y los trastornos de la personalidad. La prevalencia de diagnósticos adictológicos agudos fue mayor en comparación con los crónicos ( 2.81 \% vs. $0.80 \%)$. Se encontró que los trastornos de la personalidad del cluster B (límite, antisocial, histriónico y narcisista) fueron los más frecuentes respecto de otros trastornos de la personalidad ( $2.83 \%$ vs. $0.06 \%)$.

\section{Actividad inter-trimestral e inter-semestral}

Se observó mayor actividad del servicio los primeros semestres (enero a junio, $n=21$ 533) respecto de los segundos semestres (julio a diciembre, $n=19445$ ) durante los cinco años, encontrando $9.696 \%$ de mayor actividad en los primeros semestres respecto de los segundos semestres $(t=2783, p=0.009)$. La mayor parte de los diagnósticos psiquiátricos mostró un patrón de mayor actividad durante los primeros trimestres, la cual fue decreciendo progresivamente el resto de los trimestres; sólo los diagnósticos "Otros trastornos orgánicos" (v.gr. cambios de personalidad secundario a causa médica) y "Otros trastornos en la adolescencia" (v.gr. trastorno por tics) presentaron mayor actividad durante los segundos semestres. El trastorno depresivo mayor tuvo una mayor actividad durante los segundos trimestres. Se observó aumento de la actividad en el cuarto trimestre respecto del tercer trimestre para los diagnósticos de delirium, otros trastornos orgánicos, trastornos distímicos, trastorno de ansiedad generalizada, trastorno obsesivo compulsivo y reacciones graves al estrés, incluyendo el estrés postraumático. No se encontraron diferencias significativas inter-grupo ni intra-grupo por efecto del factor del diagnóstico psiquiátrico $(\mathrm{F}=1.459, \mathrm{p}=0.231)$. La figura 2 resume la actividad global inter-trimestral del servicio de urgencias durante el período de cinco años.

\section{DISCUSION}

Hasta donde sabemos éste es el primer estudio que describe y analiza los indicadores de actividad en un SUP mexicano en el que se detecta el total y la frecuencia relativa de consultas otorgadas al trimestre, semestre, año y quinquenio, en un servicio de urgencias de un hospital psiquiátrico de atención voluntaria sin procedimiento de triage.

El hallazgo más evidente es que se detectó un patrón de crecimiento quinquenal del $14.81 \%$ en la actividad del servicio. Aunque este fenómeno pareciera poder ser expli- 
cado secundario al incremento natural en la demanda del servicio de urgencias acorde a los fenómenos psicopatológicos y sociales actuales como aumento en el consumo de alcohol, ${ }^{33}$ el aumento en los comportamientos suicidas en los últimos años en el mundo ${ }^{34}$ y en México, ${ }^{35}$ y el incremento de conductas violentas asociadas al uso de sustancias, ${ }^{36}$ existen otros factores adicionales que pueden aumentar los indicadores de actividad de un servicio de urgencias.

Entre los factores intrínsecos a los procedimientos en los servicios de urgencias que pueden explicar el aumento de la actividad se encuentra a la presencia de usuarios frecuentadores en el servicio ${ }^{37}$ y la demanda recurrente de condiciones no urgentes. ${ }^{38}$ Ambas condiciones fueron detectadas en el SUP debido a los resultados en las variables de utilización del servicio durante el quinquenio como el incremento de usuarios sin adherencia a consulta externa (15\%), el incremento de usuarios derivados a servicios ambulatorios $(4 \%)$, y la mayor proporción de usuarios que manifestaron como principal motivo la exacerbación de síntomas psiquiátricos pre-existentes (53\%), lo anterior apoya que el tipo de utilización de servicio que se vio incrementado fue principalmente de los usuarios con motivos de consulta no urgentes ya que el envío a atención ambulatoria (Consulta externa o Preconsulta) corresponde generalmente a usuarios estables, no críticos, con demanda individual subjetiva de ameritar atención inmediata e intervención del SUP.

El crecimiento en "bola de nieve", en los indicadores actividad del SUP describe un fenómeno que podría ser atribuido a un número creciente de pacientes frecuentadores del servicio. El fenómeno del paciente frecuentador se desarrolla debido a una retroalimentación negativa entre la falta de adherencia a los servicios ambulatorios y la frecuentación del servicio de urgencias, lo cual empeora al existir una oferta de atención voluntaria sin clasificación por severidad o riesgo (triage). Lo anterior genera un fenómeno "revolvente" de usuarios frecuentadores cada vez mayor que satura más el servicio y limitan la oferta de atención a usuarios con condiciones verdaderamente urgentes.

Un hallazgo interesante es que se observó un incremento del 5\% en la actividad del SUP en el turno matutino (08:00 a 14:00 horas), así como un incremento del 10\% en el horario nocturno (20:00 a 08:00 horas del día siguiente) durante el quinquenio. Un fenómeno del que nos pudimos percatar es que un número considerable de usuarios frecuentaron el turno nocturno para evadir los procedimientos administrativos presentes durante los días y horarios hábiles, especialmente aquellos relacionados con el pago diferido de los servicios; lo anterior podría ser una explicación del sobrecargado incremento de la demanda nocturna del servicio.

Se ha visto que la oferta indiscriminada de atención de SUP a condiciones no urgentes genera diferentes problemas como sobresaturación y aumento de frecuentadores del servicio, especialmente si no existen alternativas diferentes como el envío a primer nivel en corto tiempo o integrar a programas de seguimiento de trastornos específicos. ${ }^{39}$ Lejos de aislarse de los servicios de urgencias, los servicios ambulatorios deben estar en estrecho contacto con ellos de tal forma que en ambos servicios se trabaje coordinada y sinérgicamente para incrementar la continuidad de la atención en los servicios ambulatorios, y al mismo tiempo disminuir la frecuentación de los servicios de urgencias.

Un error frecuente es afirmar que los servicios de urgencias deben operar como "amortiguadores" de la falta de oferta asistencial, especialmente cuando otros servicios no están disponibles. ${ }^{40}$ Esta tendencia a delegar a los servicios de urgencias todas las situaciones no contempladas por otros servicios podría ser una explicación de la saturación a lo largo del tiempo y en ciertas temporadas o incluso horarios de los servicios de urgencias. Lo anterior empeora si los criterios de atención en urgencias se basan en una atención voluntaria y no clasificada ni estratificada, generando progresivamente mayores problemas de sobresaturación y frecuentación en forma continua a lo largo del tiempo, ${ }^{41}$ perdiendo el servicio de urgencias su objetivo primordial que es priorizar y estabilizar las condiciones más graves ante la demanda excesiva de usuarios con urgencias sentidas, que, paradójicamente, suelen tener la percepción de que realmente ameritan una atención inmediata. ${ }^{42}$

Algunos estudios han reportado que las condiciones que se presentan en forma más frecuente visitan el servicio de urgencias fuera del horario hábil. ${ }^{43}$ lo cual confirma que la atención de un SUP debe de mantenerse las 24 horas incluso si la frecuencia relativa es menor respecto de los días y horarios hábiles.

Los resultados de este estudio mostraron que algunos diagnósticos psiquiátricos, como las condiciones adictológicas agudas y los trastornos de la personalidad del cluster $B$, aumentaron su frecuencia relativa anual durante el quinquenio. Esto es congruente con reportes de que otros factores que podrían influir en la frecuentación de servicios de urgencias son los diagnósticos adictológicos y los trastornos de la personalidad del cluster B, según el DSM-IV. ${ }^{44,45}$

Entre otros factores más que pueden favorecer la afluencia de pacientes frecuentadores de los SUP está la sensación de privacidad y de satisfacción al utilizar el servicio de urgencias. ${ }^{46}$ Otro factor de riesgo para ser frecuentador es que el usuario no alcance una percepción de mejoría en salud tras utilizar los SUP. Entre los factores relacionados con la sensación de mejoría en salud de los servicios de urgencias se encuentran la realización de triage, seguimiento por parte de enfermería y valoración por diferentes médicos del staff de urgencias. ${ }^{47}$ Finalmente, otro factor que puede favorecer la frecuentación en un servicio de urgencias es la dificultad para afianzar la alianza terapéutica que se considera un importante factor en el tratamiento, especialmente en pacientes con condiciones críticas. ${ }^{48}$

Llama la atención que a pesar de que aumentó la frecuencia de diagnósticos psiquiátricos a lo largo de los años, 
se conservó la proporción de los mismos durante el período de estudio; así mismo se encontró un incremento del 9.69\% en la actividad durante el primer semestre respecto del segundo semestre, y en el primer trimestre respecto de los demás trimestres. La mayor actividad durante los primeros trimestres y semestres podría ser explicada por el principio de estacionalidad, en el que el incremento de temperatura y humedad puede aumentar la actividad de las urgencias psiquiátricas. ${ }^{49}$

El presente estudio tiene varias limitaciones. En primer lugar no muestra una comparación por edad y género debido a que la base de datos utilizada no contaba con el dato. El factor género es decisivo para la evaluación del riesgo suicida, ${ }^{50}$ por lo que será necesario realizar nuevos estudios donde sea posible comparar el impacto en los indicadores de actividad desglosados por género. Otra limitación del estudio es que la muestra dista de ser un dato representativo de la población general, ya que al tratarse de una muestra hospitalaria no se trata de una muestra probabilística. También llama la atención que el diagnóstico más frecuente en el servicio de urgencias fue el trastorno depresivo mayor, mientras que en la población general lo es la fobia específica, ${ }^{51}$ lo cual refuerza que los diagnósticos en el servicio de urgencias pueden no representar el motivo o situación crítica que se presenta al momento de solicitar atención, ya que las condiciones en urgencias psiquiátricas son entidades críticas de naturaleza sindromática que a la fecha no están suficientemente categorizadas, por lo que los diagnósticos nosológicos actuales son insuficientes para clasificarlas adecuadamente. Una tercera limitación es que el estudio no incluyó indicadores de calidad (por ejemplo, tiempos de espera), indicadores de satisfacción (por ejemplo, conformidad con la información recibida por el médico), ni indicadores de continuidad (por ejemplo, seguimiento longitudinal de los servicios utilizados después de la última consulta de urgencias).

Los resultados de este estudio nos permiten concluir que la atención abierta e indiscriminada en urgencias, aunque sea bien intencionada y esté aparentemente centrada en la satisfacción del usuario, puede generar problemas como la sobresaturación del servicio y un aumento en los usuarios frecuentadores con condiciones no urgentes, lo cual sostiene que es necesario un procedimiento de clasificación inicial de la gravedad o riesgo de urgencias psiquiátricas, ya que la pronta identificación de las condiciones no urgentes son prioritarias para cuestiones de calidad y satisfacción en la utilización de servicios de urgencias. ${ }^{52}$ También nos permiten concluir que la atención voluntaria e indiscriminada en urgencias tiene un efecto de incremento en el volumen asistencial, gran parte del cual está formado por frecuentadores de servicios, lo cual afecta su funcionamiento global, la satisfacción del usuario $\mathrm{y}$, lo que es más serio: el trato no priorizado y desfavorable hacia las condiciones verdaderamente urgentes.

Se necesitan nuevos estudios que determinen el impacto de programas de clasificación de urgencias psiquiátricas (triage) con instrumentos o escalas específicas para clasifica- ción de urgencias en salud mental en servicios de urgencias de hospitales psiquiátricos y generales.

\section{REFERENCIAS}

1. Barton GM. The history of emergency psychiatry: A personal account. En: Glick LR, Berlin JS, Fishkind AB, Zeller S (eds). Emergency psychiatry, principles and practice. Philadelphia: Lippincott Williams \&Wilkins; 2008.

2. Bruffaerts $R$, Sabbe $M$, Demyttenaere $K$. Who visits the psychiatric emergency room for the first time? Soc Psychiatry Psychiatr Epidemiol 2006;41:580-586.

3. Fishkind $A B$, Berlin JS. Structure and function on psychiatric emergency services. En: Glick LR, Berlin JS, Fishkind AB, Zeller S (eds). Emergency psychiatry, principles and practice. Philadelphia: Lippincott Williams \& Wilkins; 2008.

4. Weissberg P. Emergency psychiatry: A critical educational omission. Ann Intern Med 1991;114:246-247.

5. Yule W. Theory, training and timing: Psychosocial interventions in complex emergencies. Intern Rev Psychiatry 2006;18(3):259-264.

6. Damsa C, Hummel C. Economic impact of crisis intervention in emergency psychiatry: A naturalistic study. Eur Psychiatry 2005;20:562-566.

7. Damsa C, Lazignac C, Cailhol L et al. Troubles dépressifs majeurs et urgences psychiatriques: Une étude naturaliste concernant la place des interventions de crise. Can J Psychiatry 2005;50:857-862.

8. Clarke P, Hafner J, Holme G. The brief andmission unit in emergency psychiatry. J Clin Psychol 1997;53(8):817-823.

9. Bruffaerts R, Sabbe M, Demyttenaere K. Predicting aftercare in psychiatric emergencies. Soc Psychiatry Psychiatr Epidemiol 2005;40:829834.

10. Carpenter L, Schecter J, Underwood J. Service expectations and clinical characteristics of patients receiving psychiatric emergency services. Psychiatr Serv 2005;56(6):743-745.

11. Way BB. Relationships between patient, family and significant other disposition preferences in psychiatric emergency services and the clinical symptom ratings and disposition decisions of psychiatrists. Psychiatr Rehab J 2005;29(2):132-137.

12. Borges G, Mondragón L, Cherpitel C, Ye Y et al. El consumo de bebidas alcohólicas y los servicios de urgencias: estudios realizados por el Instituto Nacional de Psiquiatría Ramón de la Fuente 1986-2003. Salud Mental 2003;26(5):19-27.

13. López JL, Rozovsky H. El papel que desempeña el alcohol en los motivos por los que se les da atención en los servicios de urgencia, y estimación del riesgo asociado en los traumatismos. Salud Mental 1998;21(3):32-38.

14. Borges G, Medina-Mora ME, Cherpitel C, Casanova L et al. Consumo de bebidas alcohólicas en los pacientes de los servicios de urgencias de la ciudad de Pachuca, Hidalgo. Salud Publica Mex 1999;41(1):3-11.

15. López-Jiménez JL. Patrón de consumo de alcohol en pacientes captados en salas de urgencias. Salud Publica Mex 1998;40(6):487-493.

16. Mondragón L, Saltijeral MT, Borges G, Bimbela A. La ideación suicida y su relación con la desesperanza, el abuso de drogas y alcohol. Salud Mental 1998;21(5):20-27.

17. Mondragón L, Romero M, Borges G. Ethnography in an emergency room: evaluating patients with alcohol consumption. Salud Publica Mex 2007;50(4):308-315.

18. Borges G, Orozco R, Cremonte M, Bizie-Figlie $\mathbf{N}$ et al. Alcohol and violence in the emergency departament: a regional report from the WHO collaborative study on alcohol and injuries. Salud Publica Mex 2008;50(Sup 1):S6-S11.

19. Castilla-Puentes R, Secin R, Grau A, Galeno R et al. A multicenter study of major depressive disorder among emergency patients in Latin-America countries. Depress Anxiety 2008;25(2):199-204. 
20. Castilla-Puentes R, Secin R, Grau A, Galeno R et al. A multicenter study of bipolar disorder among emergency patients in Latin-America countries depress anxiety 2011;42(1):49-67.

21. Roman JS, Griswold KS, Smith SJ, Servoss TJ. How patients view primary care: differences by minority status after psychiatric emergency. J Cult Divers 2008;15(2):56-60.

22. Lorant V, Depuydt C, Gillain B, Guillet A et al. Involuntary commitment in psychiatric care: What makes the decision? Soc Psychiatry Psychiatr Epidemiol 2007;42:360-365.

23. Luaces $\mathrm{C}$ et al. Grupo de trabajo de indicadores de calidad de la sociedad española de urgencias de pediatría. An Pediatr (Barc) 2004;60(6):569-580.

24. Jiménez-Murillo $L$ et al. Grupo de trabajo SEMES-Insalud: Calidad en los servicios de urgencias: Indicadores de calidad. Emergencias 2001;13:60-65.

25. Snowden LR, Masland MC, Wallace NT et al. Effects on outpatient and emergency mental care of strict Medicaid early periodic screening, diagnosis and treatment enforcement. Am J Public Health 2007;97(11):1951-1956.

26. Claassen CA, Kashner MT, Gilfillan SK, Larkin GL et al. Psychiatric emergency services use after implementation of managed care in a public mental health system. Psychiatr Serv 2005;56(6):691-698.

27. Hudson CG. A predictive model of the development of national mental health systems. J Ment Health Policy Econ 2010;13(4):175-187.

28. Vatnøy TK, Fossum M, Smith N, Skettebø S. Triage Assessment of registered nurses in the emergency department. Int Emerg Nurs 2013;21(2):89-96.

29. Chang G, Weiss AP, Orav EJ, Jones JA et al. Hospital variability in Emergency department length of stay for adult patients receiving psychiatric consultation: A prospective study. Ann Emerg Med 2011;58(2):127-136.

30. INEGI.http://www.inegi.org.mx/prod_serv/contenidos/espanol/bvinegi/productos/integracion/pais/aepef/2011/Aepef2011.pdf.

31. Hillen T, Szaniecki E. Cyclic variations in demand for uot.for.hours services in child and adolescent psychiatry. Imlications for service planning. Psychiatrist 2010;34(10):427-432.

32. Rickham PP. Human experimentation code of ethics of the World Medical Association. Declaration of Helsinski. BMJ 1964;18(2):177.

33. Carballo JJ, Oquendo MA, Giner L, Sher L. Alcohol-related problems in adolescents and young adults admitted to psychiatric emergency rooms. Nord J Psychiatry 2007;61(4):310-311.

34. Jiménez-Treviño L, Sáiz-Martínez PA, Paredes-Ojanguren B. Epidemiología de los comportamientos suicidas. En: Bobes-García J, Saiz-Martinez PA, García-Portilla-Gonzalez MP, Bascarán-Fernández MT, Bousoño-García M (eds). Comportamientos suicidas. Oviedo: Grupo Ars XXI de Comunicación; 2006.

35. Borges G, Knock MK, Medina-Mora ME, Benjet C et al. The epidemiology of suicide relates in Mexico. Suicide Life Threat Behav 2007;37(6):627-640.
36. Walton MA, Cunningham RM, Chermack ST et al. Correlates of violence history among injured patients in an urban emergency department: gender, substance use and depression. J Addict Dis 2007;26(3):61-75.

37. Liu SW, Nagurney JT, Chang Y, Parry BA et al. Frequent ED users: are most visits for mental health, alcohol and drug-related complaints? Am J Emerg Med 2013;31(10):1512-1515.

38. Ryan M, Spicer M, Hyett C, Barnett P. Non-urgent presentations to a paediatric emergency department parental behaviors, expectations and outcomes. Emerg Med Australas 2005;17(5-6):457-462.

39. Kirby SE, Dennis SM, Jayasinghe UW, Harris MF. Frequent emergency attenders: Is there a better way? Aust Health Rev 2011;35(4):462-7.

40. Wand T, White K. Examining models of mental health service delivery in the emergency department. Aust N Z J Psychiatry 2007;41:784-791.

41. Eroglu SE, Toprak SN, Urgan O, Onur OE et al. Evaluation of non-urgent visits to a busy urban emergency department. Saudi Med J 2012;33(9):967-972.

42. Nelsoin J. Why patients visit emergency units rather than use primary care services. Emerg Nurse 2011;19(1):32-36.

43. Johansen IH, Morken T, Hunskaar S. Contacts related to mental illness and substance abuse in primary health care: A corss.sectional study comparing patients' use of daytime versus out-of-hours primary care in Norway. Scand J Prim Health Care 2010;28:160-165.

44. Boyer L, Dassa D, Belzeaux R, Henry JM et al. Frequent visits to a French psychiatric emergency service: diagnostic variability in psychotic disorders. Psychiatr Serv 2011;62(8):966-970.

45. Minassian A, Vike GM, Wilson MP. Frequent emergency department visits are more prevalent in psychiatric, alcohol abuse and dual diagnosis conditions tan in viral illnesses such as hepatitis and human immunodeficiency virus. J Emerg Med 2013;45(4):520-525.

46. Lin YK, Lin CJ. Factors predicting patients' perception of privacy and satisfaction for emergency care. Emerg Med J 2011;28(7):604-608.

47. Wong FK, Liu J, Chang K, Chow SK. Factors predicting improved health after emergency room visits. J Clin Nurs 2008;17(7):901-910.

48. Parada LM. Desafíos en el etsablecimiento de la alianza terapéutica en pacientes suicidas. Rev Chil Psicoanal 2013;30(2):129-134.

49. Miró-García F, Setó-iBoada S, Xifró-Collsamata A, Grau-Juaquin M et al. Factores metereológicos y Urgencias psiquiátricas. Act Esp Psiquiatr 2009;37(1):34-41.

50. Narishigue R, Kawashima Y, Otaka I, Saito T et al. Gender differences in suicide attempters: A retrospective study of precipitating factors for suicide attempts at a critical emergency unit in Japan. BMC Psychiatry 2014;14:144.

51. Medina-Mora ME, Borges G, Lara-Muñoz MC, Benjet C et al. Prevalencia de trastornos mentales y uso de servicios: Resultados de la encuesta nacional de epidemiología psiquiátrica en México. Salud Mental 2003;26(4):1-16.

52. Honigman LS, Wiler JL, Rooks S, Ginde AA. National study of non-urgent emergency department visits and associated resouces utilization. West J Emerg Med 2014;14(6):609-616.

Artículo sin conflicto de intereses 